\title{
Comparative study of computed tomography (CT) and pathological diagnosis toward mediastinal lymph node metastasis in esophageal carcinoma
}

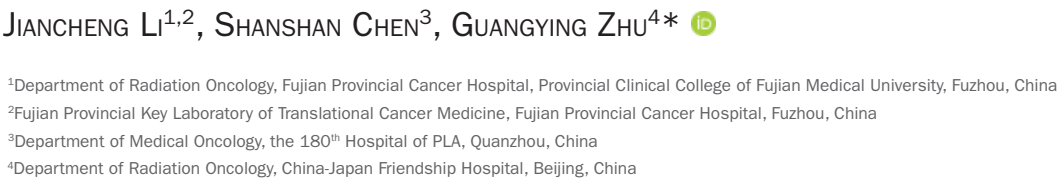

Study conducted at Fujian Provincial Cancer Hospital, Fuzhou, China

Article received: 4/24/2017 Accepted for publication: 6/26/2017

*Correspondence: Department of Radiation Oncology China-Japan Friendship Hospital Address: No. 2 East Street of Sakura Garden Chaoyang, Beijing - China Postal code: 100029 cnjianchengli@163.com

\section{SUMMARY}

Objective: To investigate the diagnostic criteria of mediastinal lymph node metastasis (MLNM) in esophageal carcinoma (EC) by comparing the lymph node sizes measured by computed tomography (CT) and obtained by postoperative pathological examination.

Method: A total of 305 EC patients were selected. MLNM location, shortest diameter and number were investigated one week before surgery, and then compared with their pathological findings.

Results: The receiver operating characteristic (ROC) curve analysis revealed that the minimum diameters of MLNM in the thoracic cavity was $8 \mathrm{~mm}$ (area under curve $[\mathrm{AUC}]=0.766$, Youden index $=0.424$ ), $5 \mathrm{~mm}$ in supraclavicular fossa (AUC $=0.785$, Youden index $=0.494), 6 \mathrm{~mm}$ in tracheoesophageal groove $(\mathrm{AUC}=0.755$, Youden index $=0.405$ ); the sensitivity was increased significantly, and the Youden index was increased significantly when compared with $10 \mathrm{~mm}$.

Conclusion: The shortest diameter of diagnostic criteria of lymph nodes in EC could be less than $10 \mathrm{~mm}$ on CT.

Keywords: Esophageal Neoplasms. Lymph Nodes. Tomography, X-ray Computed.

\section{INTRODUCTION}

Esophageal carcinoma (EC) is a common gastrointestinal cancer, with about 481,000 new cases worldwide in 2008 , and accounts for $3.8 \%$ of the total number of cancers. Its incidence has a clear regional distribution, and the morbidity and mortality of EC in developing countries account for more than $80 \%{ }^{1}$ The morbidity and mortality of EC in China ranks the first in the world. ${ }^{2}$ EC still has high incidence in China, and in 2012 it ranked the fifth of malignant tumors with its mortality raking the fourth. ${ }^{3}$ Lymph node metastasis and the number of metastases are important factors that will impact the prognosis of EC. ${ }^{4-6}$ Compared with the sixth edition, the seventh edition of tumor-node-metastasis (TNM) staging standards ${ }^{7}$ emphasizes more the impact of the number of lymph node metastasis on the staging. Presently, most Chinese and foreign scholars ${ }^{8-10}$ believe that the seventh edition of staging criteria is better than the sixth edition in evaluating the treatment options and prognosis, so, it is very important to accurately diagnose lymph node metastasis. The current standard of positive lymph node set the shortest diameter as $10 \mathrm{~mm},{ }^{11}$ but this standard is one clinical estimated value while without any pathological evidence; so, there are some controversies, because clinical metastatic lymph nodes in some parts are often smaller. This study compared the features of lymph nodes measured by $\mathrm{CT}$ and obtained by postoperative pathology, aiming to investigate the diagnostic criteria of the shortest diameter of EC-MLNM, thus providing guidance for accurate preoperative staging and outlining radiotherapeutic target areas.

\section{Method}

\section{Clinical data}

A total of 305 patients with thoracic esophageal carcinoma admitted into the Fujian Cancer Hospital from January 2012 to December 2014 were collected, including 236 males and 69 females; aging 34-82 years, with the 
mean as $58.3 \pm 8.2$ years. This study was conducted in accordance with the declaration of Helsinki. This study was conducted with approval from the Ethics Committee of Fujian Medical University. Written informed consent was obtained from all participants.

Inclusion criteria: newly diagnosed, without distant metastasis or other malignant tumor, without any anticancer treatments before surgery and performed surgery within one week of CT scanning. The basic pathological situations of the patients are shown in Table 1.

\section{Methods and parameters of CT scanning}

PHILIPS Brilliance 256-slice spiral CT scanner (Eindhoven, Holland) was used for the scanning with the parameters as: tube voltage $120 \mathrm{kV}$, tube current $300-350 \mathrm{~mA}$, scanning collimator $1 \mathrm{~mm}$, pitch 0.9 , scanning layer thickness $5 \mathrm{~mm}$, layer spacing $5 \mathrm{~mm}$, reconstruction layer thickness $2.5 \mathrm{~mm}$, and layer spacing $2 \mathrm{~mm}$. The enhanced scanning used one high-pressure syringe to rapidly inject $100 \mathrm{~mL}$ of non-ionic contrast agent (iodohydrin) from the elbow vein (injection rate $3 \mathrm{~mL} / \mathrm{s}$ ). Each patient was placed in the supine position when scanning, and the scanning area started from the supraclavicular fossa to the superior mesenteric artery level, the data of which was then transmitted into the Vitrea 2 workstation for multi-window and multi-planar reconstruction.

The classification criteria of intrathoracic lymph nodes referred to the standards revised by the American Joint Committee on Cancer-Union for International Cancer Control (AJCC-UICC) in 2009. Because partial surgical lymph node distribution methods are inconsistent with

TABLE 1 General pathological information of the patients.

\begin{tabular}{ll} 
Pathological information & $\mathbf{n}$ \\
\hline Site & \\
\hline Upper thoracic segment & 15 \\
\hline Middle thoracic segment & 206 \\
\hline Lower thoracic segment & 84 \\
\hline T staging & \\
\hline T0-1 & 57 \\
\hline T2 & 59 \\
\hline T3 & 142 \\
\hline T4 & 47 \\
\hline Pathological type & \\
\hline Poorly differentiated SqCa & 25 \\
\hline Moderately differentiated SqCa & 254 \\
\hline Highly differentiated SqCa & 15 \\
\hline Other & 11 \\
\hline
\end{tabular}

imaging methods, this study was based on the CT findings and compared with the pathological findings. In order to produce a better comparison, we requested that the surgeons divided the patients into different groups according to the locations of their individual lymph nodes resected surgically, namely the supraclavicular fossa group (SCF), the tracheoesophageal groove group (TEG), the paratrachea group $(\mathrm{pT})$, the paraesophagus group $(\mathrm{pE})$, the subcarina group $(\mathrm{sC})$ and the lung hilum group (LH). Patients who could not be confirmed were excluded, and the biggest lymph node was calculated if more were in the same CT region. Other cases that cannot be concluded into corresponding areas were excluded.

\section{Imaging data acquisition}

The best window width and position were adjusted and enlarged appropriately, targeting the grouping positions of the lymph nodes. Position, shortest diameter and number of the lymph nodes in the visual field were recorded and determined jointly by two physicians to obtain a consensus as the final result; at the same, one senior physician was arranged to be in charge of the quality control.

\section{Pathological examination}

The lymph nodes were dissected by EC radical correction as well as intraoperative thoracic + abdominal or cervical + thoracic + abdominal lymph node dissection, followed by pathological examination according to the grouping.

\section{Statistical analysis}

SPSS for windows 19.0 software package was used for the data entry and analysis; the diagnostic tests used the receiver operating characteristic (ROC) curve for the analysis, and the area under the curve, corresponding sensitivity, specificity, accuracy and the Youden index were also calculated.

\section{Results}

\section{General information}

Among the 305 patients with thoracic esophageal cancer enrolled into our study, the total number of the lymph nodes found by CT and confirmed pathologically was 1,043 , including 203 with positive pathological confirmation and 840 with negative pathological confirmation. The lymph nodes of different zones are shown in Table 2.

\section{Minimum diameter for diagnosing intrathoracic MLNM}

in esophageal carcinoma-mediastinal lymph node metastasis (EC-MLNM)

ROC curve analysis revealed that the shortest diameter of lymph node $\geq 8 \mathrm{~mm}$ can be set as the best standard for 
TABLE 2 Lymph nodes found by CT in different zones $(\mathrm{mm})$.

\begin{tabular}{lllllll} 
Distribution & SCF & TEG & PT & PE & SC & LH \\
\hline Number of pathological positive & 13 & 47 & 30 & 50 & 45 & 18 \\
\hline Min short diameter of pathological positive & 3.5 & 3.0 & 4.4 & 4.2 & 4.2 & 5.2 \\
\hline Max short diameter of pathological positive & 8.0 & 14.1 & 14.1 & 16.5 & 17.2 & 15.1 \\
\hline Number of pathological negative & 40 & 132 & 191 & 146 & 245 & 86 \\
\hline Min short diameter of pathological negative & 3.0 & 3.0 & 3.0 & 3.3 & 3.5 & 3.5 \\
\hline Max short diameter of pathological negative & 9.3 & 12.8 & 17.7 & 10.1 & 22.8 & 12.4 \\
\hline
\end{tabular}

SCF: supraclavicular fossa; TEG: tracheoesophageal groove; pT: paratrachea; pE: paraesophagus; sC: subcarina; LH: lung hilum.

diagnosing intrathoracic EC-MLNM, with area under curve (AUC) as 0.766 (Figure 1A), sensitivity as $54.5 \%$, specificity as $87.9 \%$, accuracy as $82.0 \%$ and the Youden index as 0.424 . When the shortest diameter was set $\geq 10$ $\mathrm{mm}$, the sensitivity, specificity, accuracy and Youden index were $19.6 \%, 95.8 \%, 82.4 \%$ and 0.154 , respectively; when the shortest diameter was set $\geq 5 \mathrm{~mm}$, the sensitivity, specificity, accuracy, and Youden index were 93.7\%, 21.4\%, $34.2 \%$ and 0.151 , respectively.

\section{Minimum diameter for diagnosing EC-MLNM at supraclavicular fossa}

The ROC curve analysis revealed that the shortest diameter of lymph node $\geq 5 \mathrm{~mm}$ can be set as the best standard for diagnosing EC-MLNM at supraclavicular fossa, with AUC as 0.785 (Figure 1B), sensitivity as $76.9 \%$, specificity as $72.5 \%$, accuracy as $73.6 \%$, and the Youden index as 0.494 . When the shortest diameter was set $\geq 10 \mathrm{~mm}$, the sensitivity, specificity, accuracy, and Youden index were $0,1,75.4 \%$ and 0.000 , respectively.

\section{Minimum diameter for diagnosing EC-MLNM at tracheoesophageal groove}

The ROC curve analysis revealed that the shortest diameter of lymph node $\geq 6 \mathrm{~mm}$ can be set as the best standard for diagnosing intrathoracic EC-MLNM at tracheoesophageal groove, with AUC as 0.755 (Figure 1C), sensitivity as $61.7 \%$, specificity as $78.8 \%$, accuracy as $74.3 \%$ and the Youden index as 0.405 . When the shortest diameter was set $\geq 10 \mathrm{~mm}$, sensitivity, specificity, accuracy and the Youden index were $8.5 \%, 97.7 \%, 74.3 \%$ and 0.062 , respectively; when the shortest diameter was set $\geq 5 \mathrm{~mm}$, sensitivity, specificity, accuracy, and the Youden index were $72.3 \%, 65.2 \%, 67.0 \%$, and 0.375 , respectively.

\section{Discussion}

The main clinical diagnostic methods against EC currently include: ${ }^{12}$ esophageal barium contrast, chest CT scan, ultrasound or endoscopy; however, all these methods, except for CT, have obvious limitations in diagnosing esophageal carcinoma-lymph node metastasis (EC-LNM). Studies have shown that ${ }^{13,14} \mathrm{CT}$ can clearly show the existence of lymph node metastasis with high sensitivity, specificity and accuracy, so it can be used effectively to diagnose EC-LNM. Glazer et al. ${ }^{15}$ proposed for the first time in 1984 that the shortest diameter of lymph nodes on CT is much more sensitive than the long and short diameters, which can avoid spatial errors. A shortest diameter of lymph node $\geq 10 \mathrm{~mm}$ is often used as the standard of CT to diagnose LNM; however, normal and metastatic lymph nodes overlap in size, so the accuracy of this diagnostic criterion is still controversial. Clinically, CT can reveal metastatic lymph nodes with shortest diameter of less than $10 \mathrm{~mm}$. Takemura et al. ${ }^{16}$ measured the shortest diameter of metastatic lymph nodes surgically dissected from patients with esophageal squamous cell carcinoma (ESCC) and found $65 \%$ of the samples had the shortest diameter less than $1 \mathrm{~cm}$ and $27 \%$ was less than $5 \mathrm{~mm}$. A certain study has shown that ${ }^{17} 63 \%$ of EC patients have the shortest diameter of metastatic lymph nodes measuring less than $10 \mathrm{~mm}$. The results of our study showed that, compared with $10 \mathrm{~mm}$, the shortest diameter set as $8 \mathrm{~mm}$ can effectively diagnose intrathoracic lymph node metastasis, and the sensitivity was increased from $19.6 \%$ to $54.5 \%$, while the specificity and accuracy did not change much. So, $8 \mathrm{~mm}$ as the standard can exhibit more diagnostic value, and appropriately reducing the shortest diameter standard on CT toward EC-LNM is more rational.

Studies about the diagnostic criteria of CT in EC-LNM are many, while fewer studies are accompanied by pathological evidence, and recent studies just included mediastinal lymph nodes as part of their results for the sake of statistics. ${ }^{18,19}$ Our study took into account the unique features of the lymph nodes at the supraclavicular fossa and tracheoesophageal groove, and performed statistical analysis toward them, respectively. Compared with simple intrathoracic lymph node metastasis, the prognosis of the patients with thoracic esophageal carcinoma, which metastasized toward the supraclavicular fossa, was signifi- 


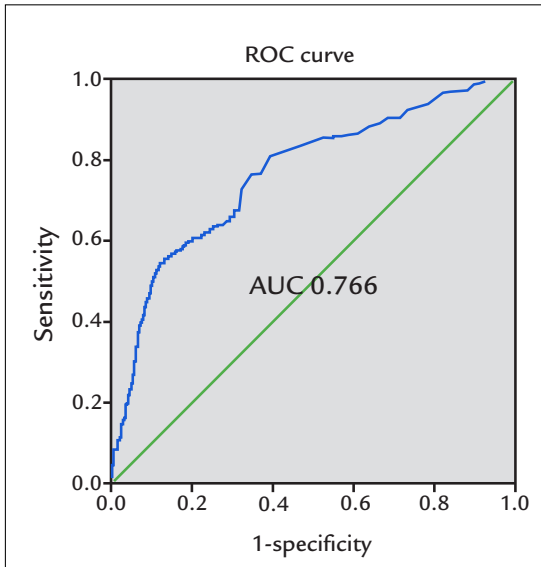

A. Intrathoracic

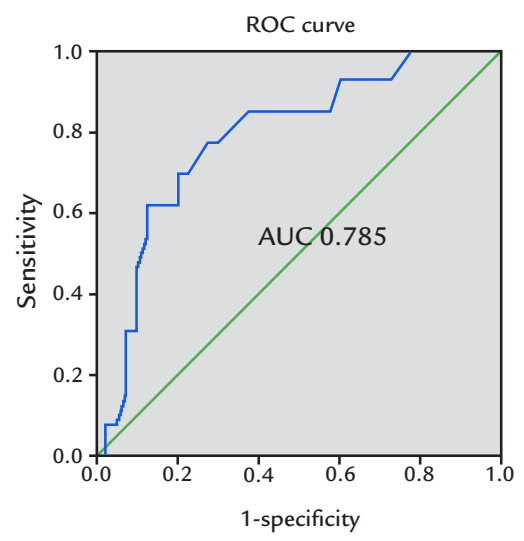

B. Supraclavicular

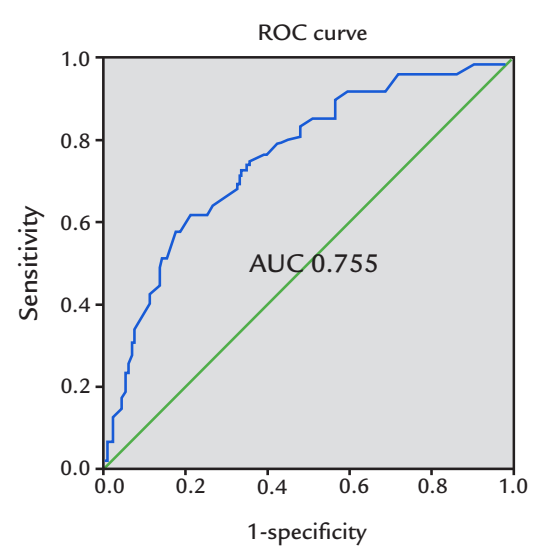

B. Tracheoesophageal groove

FIGURE 1 ROC curve of shortest diameter of lymph nodes in different areas.

cantly worse. At present, there is no related report about the CT diagnostic criteria targeting supraclavicular lymph nodes in EC. In our study, we considered that shortest diameter of EC-supraclavicular fossa LNM which can be diagnosed by CT is $5 \mathrm{~mm}$. Patients with tracheoesophageal groove lymph node metastasis can present hoarseness, drinking cough, difficulty to breath or even death by asphyxia in severe cases, so it has become an independent risk factor of death. Kato et al. ${ }^{20}$ considered that EC-tracheoesophageal groove lymph node metastasis can occur in any locations, lesions and tumor cell invasion ranges of primary tumor. Li et al. ${ }^{21}$ believed that the rate of lymph node metastasis to cervical tracheoesophageal groove and medial supraclavicular zone from middle thoracic section of esophageal cancer was increased with later $\mathrm{T}$ stages. Schmidt et al..$^{22}$ considered that general people have lymph nodes in their tracheoesophageal groove, with an average of 3.24 and 5.52 lymph nodes in the left and right tracheoesophageal grooves, respectively. Clinically, lymph nodes at the tracheoesophageal grooves are often found with a shortest diameter significantly less than $10 \mathrm{~mm}$ on CT also confirmed on pathological examination. In our study, the shortest diameter for diagnosing EC-tracheoesophageal groove lymph node metastasis was $6 \mathrm{~mm}$.

Lymphadenectasis may be caused by tumor metastasis as well as inflammatory enlargement, proliferative enlargement, or histiocytic hyperplasia-induced enlargement; some tumor cells may enter lymph nodes causing pathological features to take place despite any perceptible changes in nodal size. So, it will easily result in false-positive and false-negative conclusions if determining LNM only based on the lymph node size. ${ }^{23,24}$ In addition to the sizes on CT, metastatic lymph nodes may also reveal changes in density, edge or shape to prompt the metastasis, so how to diagnose EC-LNM with both size standards and other diagnostic methods remains a unsolved problem that needs further studies. However, clinically, the shortest diameter is more practical and intuitive to be used as the standard.

\section{Conclusion}

The shortest diameters for diagnosing MLNM in the thoracic cavity, supraclavicular fossa and tracheoesophageal groove were $8 \mathrm{~mm}, 5 \mathrm{~mm}$ and $6 \mathrm{~mm}$, respectively, and it is reasonable to reduce the $\mathrm{CT}$ diagnostic criteria of the shortest diameter of positive lymph nodes in EC.

\section{Conflict of interest}

The authors declare no conflict of interest.

\section{References}

1. International Agency for Research on Cancer. GLOBOCAN2008 [R/OL] [cited 2011 Nov 28]. Available from: http://globocan.iarc.fr/.

2. Torre LA, Bray F, Siegel RL, Ferlay J, Lortet-Tieulent J, Jemal A. Global cancer statistics, 2012. CA Cancer J Clin. 2015; 65(2):87-108.

3. Song B, Cui H, Li Y, Cheng C, Yang B, Wang F, et al. Mutually exclusive mutations in NOTCH1 and PIK3CA associated with clinical prognosis and chemotherapy responses of esophageal squamous cell carcinoma in China. Oncotarget. 2016; 7(3):3599-613.

4. Wijnhoven BP, Tran KT, Esterman A, Watson DI, Tilanus HW. An evaluation of prognostic factors and tumor staging of resected carcinoma of the esophagus. Ann Surg. 2007; 245(5):717-25.

5. Gertler R, Stein HJ, Langer R, Nettelmann M, Schuster T, Hoefler H, et al. Long-term outcome of 2920 patients with cancers of the esophagus and esophagogastric junction: evaluation of the New Union Internationale Contre Ie Cancer/American Joint Cancer Committee staging system. Ann Surg. 2011; 253(4):689-98.

6. Chan DS, Fielding P, Roberts SA, Reid TD, Ellis-Owen R, Lewis WG. Prognostic significance of 18-FDG PET/CT and EUS-defined tumour characteristics in patients with oesophageal cancer. Clin Radiol. 2013; 68(4):352-7. 
7. Edge SB, Byrd DR, Compton CC, Fritz AG, Greene FL, Trotti A, et al. AJCC cancer staging manual. 7. ed. New York: Springer; 2010.

8. Gaur P, Hofstetter WL, Bekele BN, Correa AM, Mehran RJ, Rice DC, et al. Comparison between established and the Worldwide Esophageal Cancer Collaboration staging systems. Ann Thorac Surg. 2010; 89(6):1797-803.

9. Talsma K, van Hagen P, Grotenhuis BA, Steyerberg EW, Tilanus HW, van Lanschot JJ, et al. Comparison of the 6th and 7th editions of the UICC-AJCC TNM Classification for Esophageal Cancer. Ann Surg Oncol. 2012; 19(7):2142-8.

10. Wang W, Sun XW, Li CF, Lv L, Li YF, Chen YB, et al. Comparison of the 6th and 7 th editions of the UICC TNM staging system for gastric cancer: results of a Chinese single-institution study of 1,503 patients. Ann Surg Oncol. 2011; 18(4):1060-7.

11. Bhutani MS, Hawes RH, Hoffman BJ. A comparison of the accuracy of echo features of endoscopic ultrasound (EUS) and EUS-guided fine-needle aspiration for diagnosis of malignant lymph node invasion. Gastrointest Endosc. 1997; 45(6):474-9.

12. Puli SR, Reddy JB, Bechtold ML, Antillon D, Ibdah JA, Antillon MR. Staging accuracy of esophageal cancer by endoscopic ultrasound: a meta-analysis and systematic review. World J Gastroenterol. 2008; 14(10):1479-90.

13. Liu M, Chen Y, Fu X, Zhao K, Jiang GL. Proposed revision of CT-based cervical and thoracic lymph node levels for esophageal cancer in UICC 7th version. Radiother Oncol. 2014; 113(2):175-81.

14. Sultan R, Haider Z, Chawla TU. Diagnostic accuracy of CT scan in staging resectable esophageal cancer. J Pak Med Assoc. 2016; 66(1):90-2.

15. Glazer GM, Orringer MB, Gross BH, Quint LE. The mediastinum in nonsmall cell lung cancer: CT-surgical correlation. AJR Am J Roentgenol. 1984; 142(6):1101-5.

16. Takemura M, Osugi H, Takada N, Kinoshita H, Higashino M. Prognostic factors in patients with squamous oesophageal cancer associated with solitary lymph node metastasis after esophagectomy and extended lymphadenectomy. Oncol Rep. 2003; 10(1):75-80.

17. Alper F, Turkyilmaz A, Kurtcan S, Aydin Y, Onbas O, Acemoglu H, et al. Effectiveness of the STIR turbo spin-echo sequence MR imaging in evaluation of lymphadenopathy in esophageal cancer. Eur J Radiol. 2011; 80(3):625-8.

18. Mizowaki T, Nishimura Y, Shimada Y, Nakano Y, Imamura M, Konishi J, et al. Optimal size criteria of malignant lymph nodes in the treatment planning of radiotherapy for esophageal cancer: evaluation by computed tomography and magnetic resonance imaging. Int J Radiat Oncol Biol Phys. 1996; 36(5):1091-8

19. Gao F, Li C, Gu Y, Huang J, Wu P. CT-guided 125I brachytherapy for mediastinal metastatic lymph nodes recurrence from esophageal carcinoma: effectiveness and safety in 16 patients. Eur J Radiol. 2013; 82(2):e70-5.

20. Kato H, Igaki H, Tachimori Y, Watanabe H, Tsubosa Y, Nakanishi Y. Assessment of cervical lymph node metastasis in the staging of thoracic esophageal carcinoma. J Surg Oncol. 2000; 74(4):282-5.

21. Li X, Zhao J, Liu M, Zhai F, Zhu Z, Yu F, et al. Determination of radiotherapeutic target zones for thoracic esophageal squamous cell cancer with lower cervical lymph node metastasis according to CT-images. Oncotarget. 2016; 7(24):35865-73.

22. Schmidt AF Jr, Rodrigues OR, Matheus RS, Kim JU, Jatene FB. Mediastinal lymph node distribution size and number: definitions based on an anatomical study. J Bras Pneumol. 2007; 33(2):134-40.

23. Merkow RP, Bilimoria KY, Keswani RN, Chung J, Sherman KL, Knab LM, et al. Treatment trends, risk of lymph node metastasis, and outcomes for localized esophageal cancer. J Natl Cancer Inst. 2014; 106(7).

24. Yokota T, Igaki H, Kato K, Tsubosa Y, Mizusawa J, Katayama H, et al. Accuracy of preoperative diagnosis of lymph node metastasis for thoracic esophageal cancer patients from JCOG9907 trial. Int J Clin Oncol. 2016; 21(2):283-8. 